\title{
Myomegalin is a novel A-kinase anchoring protein involved in the phosphorylation of cardiac myosin binding protein $\mathrm{C}$
}

\author{
Gerrida M Uys ${ }^{1}$, Amsha Ramburan', Benjamin Loos², Craig J Kinnear ${ }^{1}$, Lundi J Korkie', Jomien Mouton', \\ Johann Riedemann ${ }^{1}$ and Johanna C Moolman-Smook ${ }^{1 *}$
}

\begin{abstract}
Background: Cardiac contractility is regulated by dynamic phosphorylation of sarcomeric proteins by kinases such as CAMP-activated protein kinase A (PKA). Efficient phosphorylation requires that PKA be anchored close to its targets by A-kinase anchoring proteins (AKAPs). Cardiac Myosin Binding Protein-C (CMyBPC) and cardiac troponin I (CTNI) are hypertrophic cardiomyopathy (HCM)-causing sarcomeric proteins which regulate contractility in response to PKA phosphorylation.

Results: During a yeast 2-hybrid $(\mathrm{Y} 2 \mathrm{H})$ library screen using a trisphosphorylation mimic of the C1-C2 region of CMyBPC, we identified isoform 4 of myomegalin (MMGL) as an interactor of this $\mathrm{N}$-terminal CMyBPC region. As MMGL has previously been shown to interact with phosphodiesterase $4 \mathrm{D}$, we speculated that it may be a PKAanchoring protein (AKAP).

To investigate this possibility, we assessed the ability of MMGL isoform 4 to interact with PKA regulatory subunits R1A and R2A using Y2H-based direct protein-protein interaction assays. Additionally, to further elucidate the function of MMGL, we used it as bait to screen a cardiac CDNA library. Other PKA targets, viz. CARP, COMMD4, ENO1, ENO3 and CTNI were identified as putative interactors, with cTNI being the most frequent interactor. We further assessed and confirmed these interactions by fluorescent 3D-co-localization in differentiated H9C2 cells as well as by in vivo co-immunoprecipitation. We also showed that quantitatively more interaction occurs between MMGL and CTNI under $\beta$-adrenergic stress. Moreover, siRNA-mediated knockdown of MMGL leads to reduction of CMyBPC levels under conditions of adrenergic stress, indicating that MMGL-assisted phosphorylation is requisite for protection of cMyBPC against proteolytic cleavage.

Conclusions: This study ascribes a novel function to MMGL isoform 4: it meets all criteria for classification as an AKAP, and we show that is involved in the phosphorylation of CMYBPC as well as CTNI, hence MMGL is an important regulator of cardiac contractility. This has further implications for understanding the patho-aetiology of HCM-causing mutations in the genes encoding CMYBPC and CTNI, and raises the question of whether MMGL might itself be considered a candidate HCM-causing or modifying factor.
\end{abstract}

\section{Background}

Cardiac contractility is significantly enhanced by the dynamic phosphorylation of a number of sarcomeric proteins, including cardiac myosin binding protein $\mathrm{C}$ (cMyBPC) $[1,2]$. This highly modular protein, found in the C-zone of the sarcomere, is encoded by a gene

\footnotetext{
* Correspondence: hm@sun.ac.za

'US/MRC Centre for Molecular and Cellular Biology, Department of

Biomedical Sciences, University of Stellenbosch, South Africa

Full list of author information is available at the end of the article
}

which is frequently implicated in hypertrophic cardiomyopathy (HCM) [3], a common inherited cardiac disease characterised by hypertrophy of the ventricular muscle [4]. There are multiple isoforms of this protein; the cardiac isoform differs from its skeletal counterparts by containing an extra immunoglobulin-like (IgI) domain $(\mathrm{C} 0)$ at the amino terminal, a charged residuerich insertion in domain $\mathrm{C} 5$ and three phosphorylation sites in a motif between the second and third IgI domains (C1-C2), known as the MyBPC motif or m-

\section{C) Biomed Central}


domain. Originally thought to have only a structural role, cMyBPC has been shown to play an important role in the regulation of cardiac contractility [1], for which the N-terminal region of the protein appears to be crucial. Upon $\beta$-adrenergic stimulation, three sites within the MyBPC motif are phosphorylated by protein kinase A (PKA) and calcium/calmodulin-activated protein kinase $(\mathrm{CaMK})$, the phosphorylation of this region of MyBPC then leads to rearrangement of the myosin crossbridges and thick filament structure $[1,5,6]$ such that cardiac contractility is increased [7].

However, since these kinases regulate a broad range of cellular responses, their compartmentalization in close proximity to their sarcomeric targets is required to facilitate control over which proteins are phosphorylated in response to second messenger signalling [8,9]. At the same time, co-compartmentalization of enzymes or proteins that generate or terminate these second messenger metabolites, such as the phosphodiesterases (PDEs) which degrade cAMP and cGMP, with the relevant responsive kinases helps to optimise the precision and speed of response to second messenger signaling [10]. Compartmentalization of kinases in general is achieved by either direct docking of the kinase on the target protein, or by anchoring of the kinase to, or close to, the target via an adaptor protein, named A-kinase anchoring proteins or AKAPs in the case of PKA [11]. Although a CaMK has been found to co-purify with cMyBPC $[1,12]$, the mechanism of co-compartmentalization of cMyBPC and PKA has never been described and very little is known about sarcomeric AKAPs in general.

In this study we identified myomegalin (MMGL) isoform 4, a PDE4D-interacting protein [13], as a binding partner of PKA, the cMyBPC N-terminal region, as well as other PKA-targets, and show that MMGL meets all the requirements for classification as a novel sarcomeric AKAP, with important implications for regulation of cardiac contractility during adrenergic stimulation.

\section{Results}

Interaction of trisphospho-cMyBPC with MMGL

As the interactions of the $\mathrm{N}$-terminal region of $\mathrm{cMyBPC}$ under its various phosphorylation states appear to be integral to the regulatory role of $\mathrm{cMyBPC}$ in contractility, we aimed to gain further insight into the interactions of the trisphosphorylated form of the N-terminal region of $\mathrm{cMyBPC}$. Thus, we used a construct of $\mathrm{C} 1-\mathrm{C} 2$ in which the serines in the three phosphorylation sites of the MyBPC motif had been replaced by glutamic acids (PPP), mimicking the trisphosphorylated state, as bait in a $\mathrm{Y} 2 \mathrm{H}$ cardiac library screen.

During successive rounds of nutritional and colorimetric selection, 19 putative interactors of PPP were identified, which were able to activate the HIS3, ADE2 and MEL1 reporter genes in the presence of the PPP bait, but not in the presence of heterologous baits (Table 1). Of these, three in-frame prey plasmids encoded isoform 4 of PDE4D-interacting protein, also known as myomegalin (MMGL) (Table 1). This putative interaction between MMGL and the N-terminal of cMyBPC was intriguing, as the former protein is known to anchor PDE4D to particulate structures; PDE4D, in turn, is known to hydrolyze cAMP and thus to attenuate PKA-driven phosphorylation [13]. Furthermore, it has been shown that some adaptor proteins can anchor both PKA and PDE4D [14], raising the possibility that MMGL may be anchoring PKA to at least cMyBPC in the sarcomere as an AKAP; hence this interaction was prioritized for further investigation.

3D-fluorescence microscopy indicated that cMyBPC and MMGL isoform 4 colocalize in the cytoplasmic (encompassing the sarcomeric) region of differentiated rat cardiac H9C2 cells (Figure 1A). Exposure of the cells to the adrenergic agonist isoproterenol led to an increase in co-localization of the two proteins, as evidenced by the increased yellow staining during fluorescence microscopy of such cells (Figure 1B, C).

Furthermore, in order to verify that it is the C1-C2 region of $\mathrm{CMyBPC}$ that interacts with MMGL isoform 4, specifically, and does so in the absence of the GAL4 regions of the $\mathrm{Y} 2 \mathrm{H}$ bait protein, we used in vitro coimmunoprecipitation assays. We also used these assays to assess whether trisphosphorylation of the MyBPC motif within this region was crucial to the putative interaction. Both the native $\mathrm{C} 1-\mathrm{C} 2$ and a trisphosphomimic of the $\mathrm{C} 1-\mathrm{C} 2$ region interacted with MMGL isoform 4 in the absence of the Y2H GAL4 domains (Figure 2A), suggesting that the interaction can take place whether or not the MyBPC-motif is phosphorylated. Interaction between $\mathrm{CMyBPC}$ and MMGL isoform 4 was further confirmed in a cellular environment by reciprocal co-immunoprecipitation of these tagged proteins in $\mathrm{H} 9 \mathrm{C} 2$ cells (Figure 2B).

\section{MMGL binds to PKA regulatory subunits}

As the primary characteristic of an AKAP is the ability to bind at least one PKA regulatory subunit, we investigated the possibility that MMGL functions as an AKAP by using $\mathrm{Y} 2 \mathrm{H}$-based direct protein-protein interaction assays to determine binding between MMGL and the two cardiac-expressed regulatory subunits of PKA. Both regulatory subunits (PRKAR1A and PRKAR2A) interacted with MMGL, as assessed by growth and light colour of the colonies on quadruple dropout nutritional selection medium, compared to relevant controls (Figure 3A).

Further support for this finding was garnered by colocalization studies using 3D-fluorescence microscopy: 


\begin{tabular}{|c|c|c|c|}
\hline Clone \# & $\begin{array}{l}\text { Genomic Hit, Accession no } \\
\text { (E-value) }\end{array}$ & In-frame ORF Protein Hit, Accession no (E-value) & Subcellular localization \\
\hline $\begin{array}{l}\text { P205, P272, } \\
\text { P403 }\end{array}$ & $\begin{array}{l}\text { PDE4DIP, transcript } 4 \\
\text { NM_001002810 (0.0) }\end{array}$ & $\begin{array}{l}\text { Homo sapiens phosphodiesterase 4D interacting protein } \\
\text { (myomegalin) NP_055459.3 (1e-70) }\end{array}$ & Cytoplasm, Cytoskeleton \\
\hline $\begin{array}{l}\text { P182, P286, } \\
\text { P267 }\end{array}$ & ACTC1 NM_005159 (0.0) & Homo sapiens actin, alpha, cardiac muscle 1 NP_005150 (1e-89) & Thin filament sarcomere \\
\hline P259 & ACTCB NM_001101 (0.0) & Homo sapiens actin, beta $\underline{\mathrm{AAH} 23548.1(8 \mathrm{e}-34)}$ & Cytoplasm, cytoskeleton \\
\hline $\begin{array}{l}\text { P62, P213, } \\
\text { P319 }\end{array}$ & MYBPC3 NM_000256 (0.0) & $\begin{array}{l}\text { Homo sapiens cardiac myosin binding protein C, NP_000247.2 (2e- } \\
\text { 26) }\end{array}$ & Thick filament; Sarcomere \\
\hline $\mathrm{P} 2, \mathrm{P} 81$ & $\begin{array}{l}\text { Gcom1 transcript } 12 \\
\text { NM_001018100 (0.0) }\end{array}$ & Homo sapiens GRINL1A combined protein NP_001018100.1, (2e-65) & Unknown \\
\hline P80 & FLNC NM_001458.2 (0.0) & Homo sapiens filamin C, gamma NP_001449.3 (2e-91) & $\begin{array}{l}\text { Myofibrillar z-disks; actin } \\
\text { cytoskeleton }\end{array}$ \\
\hline P175, P465 & CNN1 NM_001299.4 (0.0) & $\begin{array}{l}\text { Homo sapiens calponin 1, basic, smooth muscle NP_001290.2 (1e- } \\
\text { 104) }\end{array}$ & Cytoplasm, cytoskeleton \\
\hline P11 & PARD3 NM_019619.2 (3e-121) & $\begin{array}{l}\text { Homo sapiens par-3 partitioning defective } 3 \text { homolog } \\
\text { NP_062565.2 (1e-24) }\end{array}$ & Plasma membrane; tight junctions \\
\hline P223 & CCT7 NM_001009570.1 (0.0) & $\begin{array}{l}\text { Homo sapiens chaperonin containing TCP1, subunit } 7 \text { (eta) } \\
\text { NP_001009570.1 (1e-83) }\end{array}$ & Cytoplasm \\
\hline P238 & SVIL NM_021738.1 (0.0) & Homo sapiens supervillin NP_003165.1 (1e-15) & $\begin{array}{l}\text { Actin cytoskeleton, plasma } \\
\text { membrane, nucleus }\end{array}$ \\
\hline P398 & FLJ21347 NM_022827.2 (0.0) & FLJ21347 NP_073738.2 (4e-101) & Unknown \\
\hline
\end{tabular}

we demonstrated that MMGL isoform 4 occurs in the same 3D-subcellular region as the two PKA regulatory isoforms in H9C2 cardiomyocytes (Figure 3B).

To further verify physical interaction between MMGL and these PKA isoforms in a cellular context and in the absence of GAL4 domains, we performed pull-down assays in differentiated $\mathrm{H} 9 \mathrm{C} 2$ cardiomyocytes. As commercial antibodies against MMGL/PDE4DIP are not able to detect isoform 4, the smallest isoform of this protein, we used an antibody directed against dsRed to detect a dsRed-tagged version of MMGL isoform 4 in these assays. In this way, endogenous PRKAR1A and PRKAR2A were shown to immunoprecipitate dsRedMMGL, and vice versa (Figure 3C), indicating physical interaction of MMGL with these two PKA regulatory isoforms.

\section{MMGL binds to additional PKA targets}

We further investigated the function of MMGL isoform 4 by using it as $\mathrm{Y} 2 \mathrm{H}$ bait to screen a cardiac cDNA library in order to identify its additional binding partners. Thirteen in-frame putative MMGL-interactors were identified that activated all three nutritional reporter genes in the presence of the MMGL bait, but not in the presence of heterologous baits (Table 2). As we were primarily interested in the possibility of MMGL acting as a sarcomeric AKAP, proteins with defined vesicular localizations were not considered of primary interest for follow-up in this study; these included the mitochondrial protein COX5A, the proteosome $26 \mathrm{~S}$ subunit and the endosomal protein SNX3. Of the remaining ten putative MMGL interactors, six encoded cardiac troponin I (cTNI) (Table 2).

Further support for the validity of these interactions was provided by finding that MMGL occurs in the same $3 \mathrm{D}$-subcellular region as all five of the putative interactors identified in the $\mathrm{Y} 2 \mathrm{H}$ library screen, viz. cardiac ankyrin repeat protein (CARP), copper metabolism gene MURR1 domain 4 (COMMD4), $\alpha$-enolase (ENO1), $\beta$ enolase (ENO3) (Figure 4), and cardiac troponin I (cTNI) (Figure 5), in differentiated cardiomyocytes.

Moreover, in pull-down assays, exogenous, fluorescently-tagged MMGL and endogenous ENO1, ENO3, CARP and cTNI reciprocally co-precipitated each other (Figure 6i-iv). As COMMD4 had a similar mobility to antibody light chains, which interfered with detection of these proteins in Western blots, a GFP-tagged fusion of this protein was expressed in $\mathrm{H} 9 \mathrm{C} 2$ cells for pull-down assays. In these assays, exogenous GFP-COMMD4 immunoprecipitated exogenous dsRed-MMGL, and vice versa (Figure 6v). Thus, Western blot analysis data supported the proposed interaction of MMGL with ENO1, ENO3, CARP, cTNI and COMMD4.

cTNI is a known PKA target [15], while the remaining four putative interactors were shown to be likely targets using Phosphomotif Finder http://www.hprd.org/PhosphoMotif_finder; we therefore investigated the effect of isoproterenol stimulation of the $\mathrm{H} 9 \mathrm{C} 2$ cells on co-localization, using the most frequent, and sarcomeric-located, putative interactor, cTNI, as example. Treating H9C2 
A)

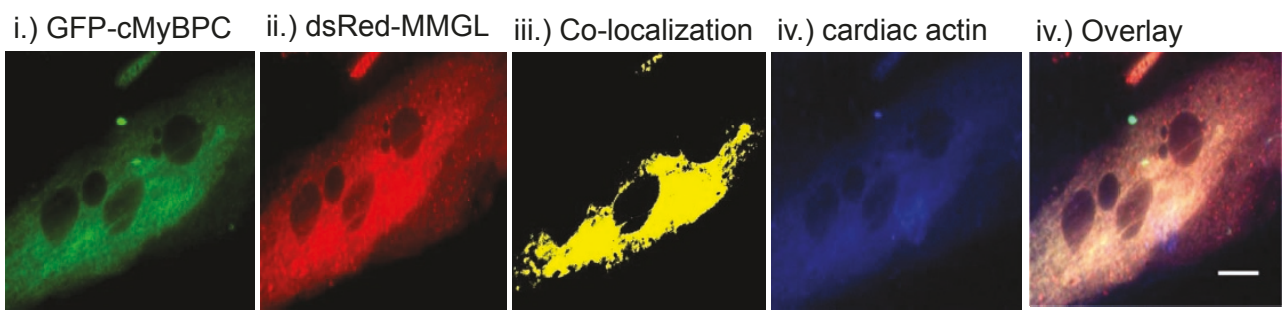

B)

i.) GFP-cMyBPC

- isopro

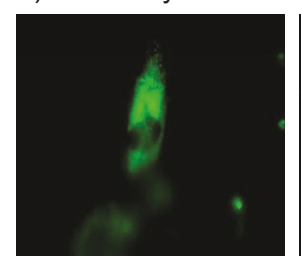

+ isopro

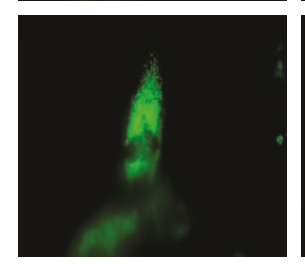

iv.) Overlay with

Hoechst-stained

ii.) dsRed-MMGL

iii.) Co-localization

nuclei
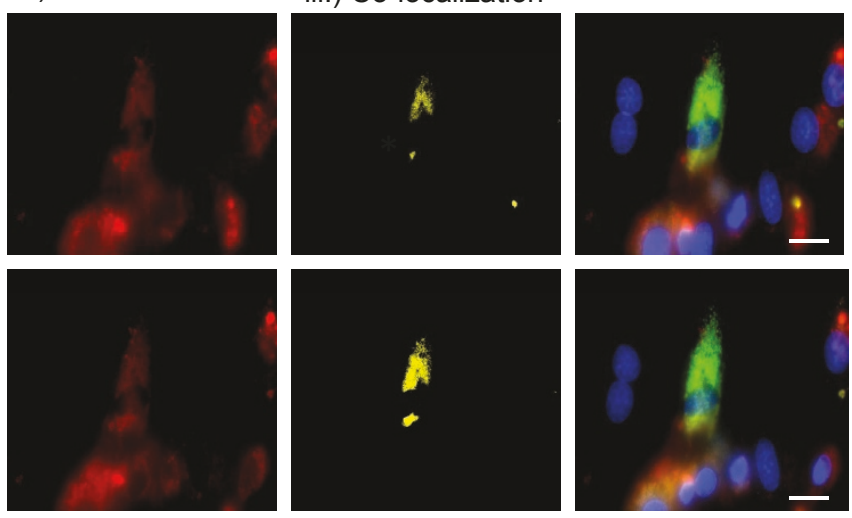

C)

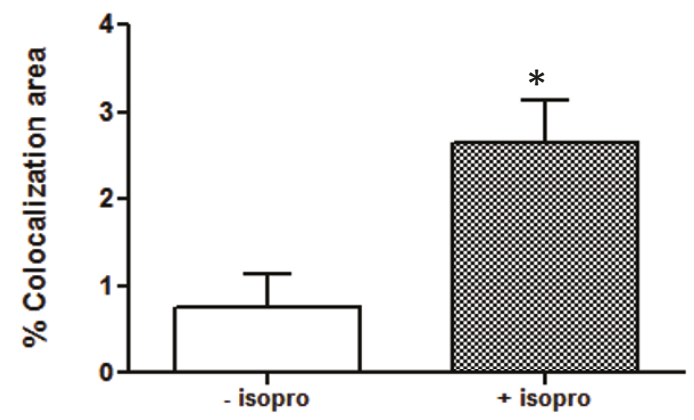

Figure 1 MMGL isoform 4 interacts with the C1-C2 region of cMyBPC. A. Representative image of live cell fluorescence microscopy showing co-localization of CMyBPC and MMGL isoform 4. Each panel represents a single frame of the 25 images that were captured for the vertical Z-stack. The first four panels shows a single colour channel, while the image in the last panel shows an overlay of the four colour channels used. Column (iii) shows co-localization (yellow fluorescence) between dsRed-MMGL and GFP-cMyBPC, while column (iv) shows cardiac actin, a marker of the sarcomeric region. Scale bar: $0.02 \mathrm{~mm}$. B. Representative image of live cell fluorescence microscopy showing that colocalization of MMGL isoform 4 and CMyBPC increases under adrenergic stress. Each panel represents a single frame of the 25 images that were captured for the vertical Z-stack. Each of the first three columns shows a single colour channel, while the image in the last column shows an overlay of the four colour channels used. Column (iii) shows co-localization (yellow fluorescence) between dsRed-MMGL and GFP-cMyBPC in the absence (-isopro) and presence (+isopro) of the beta-adrenergic agonist, isoproterenol. As evidenced by the increased yellow staining, colocalization levels between MMGL and CMyBPC increased ten minutes after the addition of isoproterenol. Scale bar: $0.02 \mathrm{~mm}$. C. Quantification of co-localization shown in B demonstrates the significant increase in co-localization after the addition of isoproterenol $\left( \pm \operatorname{SEM},{ }^{*} p<0.05, n=5\right)$. Change in co-localization was calculated using the CellR software and presented as a false colour image and percent co-localization as described by Loos et al., 2008 [29]. Abbreviations: isopro = isoproterenol 


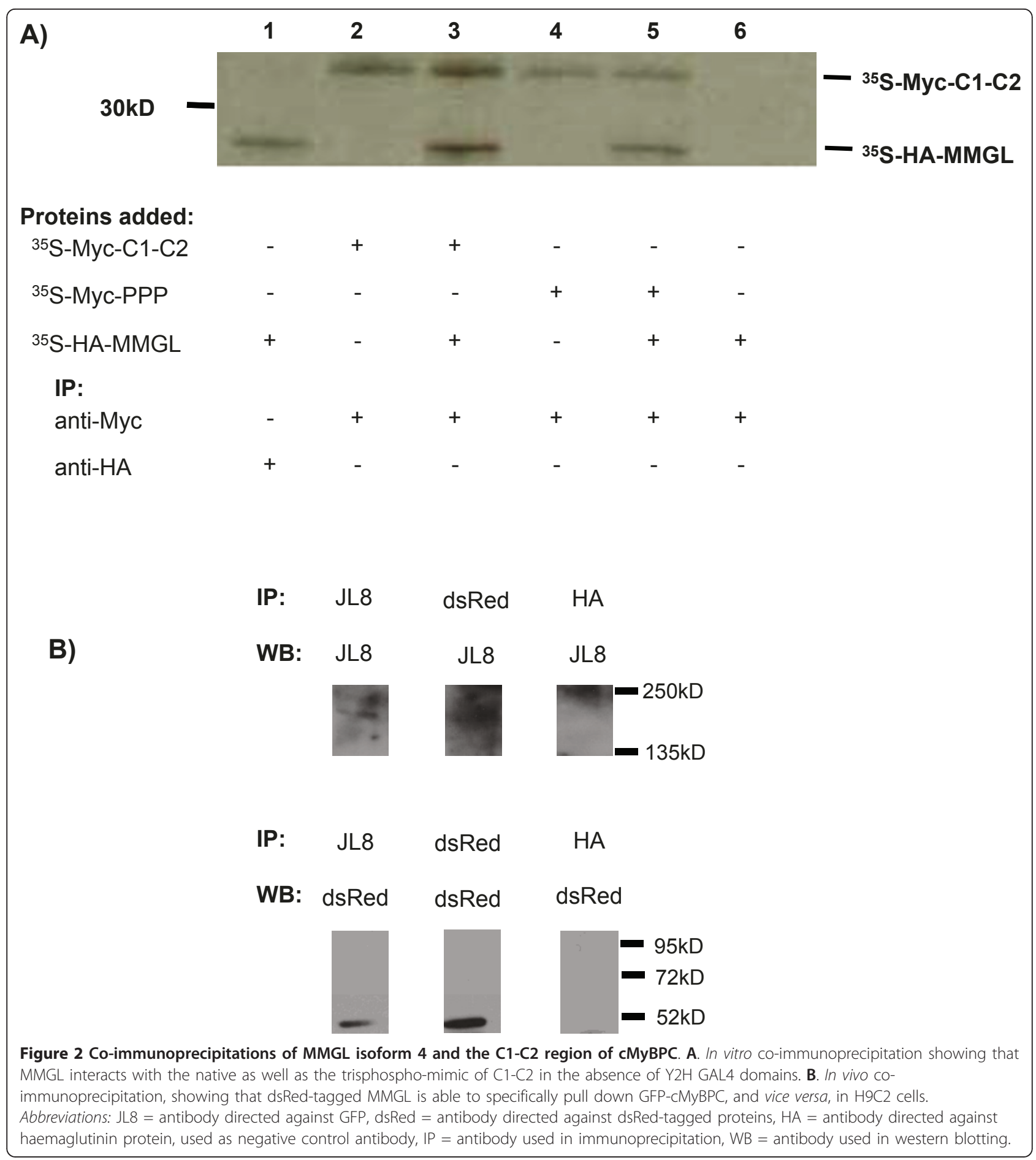

cells transfected with dsRed-MMGL/GFP-cTNI with isoproterenol resulted in significantly more 3D co-localization between these two proteins, as shown in Figure $5 \mathrm{~B}$ and $5 \mathrm{C}$, compared to non-treated cells.

Thus, our results strongly suggest that MMGL interacts with both PKA regulatory isoforms, as well as with each of the prioritized five putative prey interactors identified in the $\mathrm{Y} 2 \mathrm{H}$ screen, in a cellular milieu, and in the absence of the GAL4 domains.

\section{Effect of MMGL knockdown}

In order to evaluate the role of MMGL in CMyBPC phosphorylation, we assessed the expression of the different phosphorylation isoforms of $\mathrm{cMyBPC}$, in the 


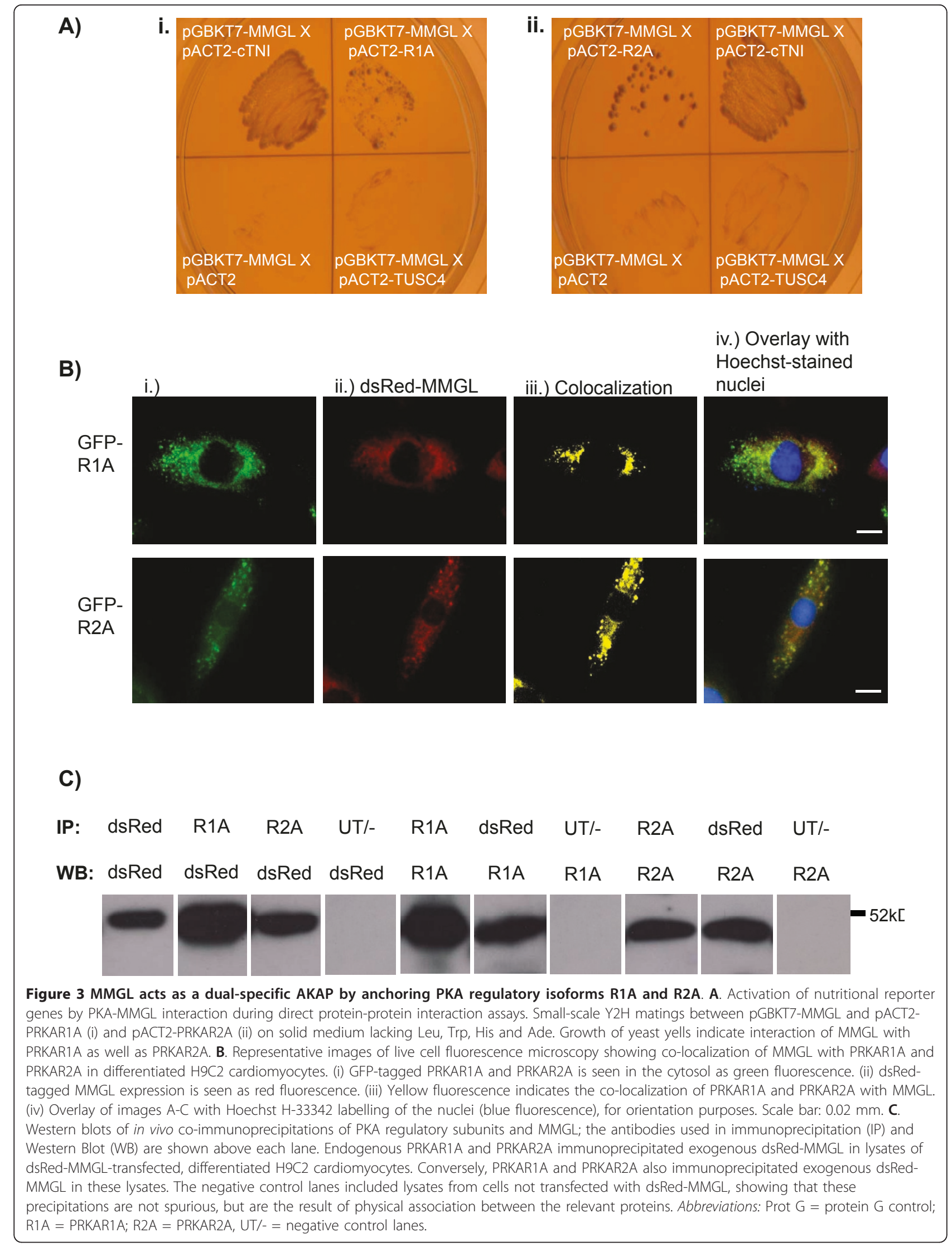


Table 2 Interactors of MMGL isoform 4 identified by yeast 2-hybrid library screening

\begin{tabular}{llll}
\hline Clone \# & $\begin{array}{l}\text { Genomic Hit, Accession no \& E- } \\
\text { value }\end{array}$ & In-frame ORF Protein Hit, Accession no \& E-value & $\begin{array}{c}\text { Subcellular } \\
\text { localization }\end{array}$ \\
\hline $128,137,148,149,160$, & TNNI3 NM 000363, 0.0 & $\begin{array}{l}\text { Homo sapiens troponin I type 3 (cardiac) NP 000354.3, 2e- } \\
82\end{array}$ & Thin filament \\
200 & CARP NM 014391, 0.0 & $\begin{array}{l}\text { Homo sapiens Cardiac ankyrin repeat protein NP 055206, } \\
\text { 2e-121 }\end{array}$ & Cytosol, nucleus \\
192 & Homo sapiens COMM domain containing 4 NP 060298.2, & Cytoplasm \\
715 & COMMD4 NM 017828, 0.0 & $\begin{array}{l}\text { 3e-97 } \\
\text { Homo sapiens Enolase 1 (alpha) NP 001419, 4e-116 }\end{array}$ & Cytoplasm \\
130 & ENO1 NM 001428, 0.0 & Homo sapiens Enolase 3 (beta, muscle) NP 001967.1, 000.1 Cytoplasm & Cys \\
203 & ENO3 NM 053013, 0.0 & &
\end{tabular}

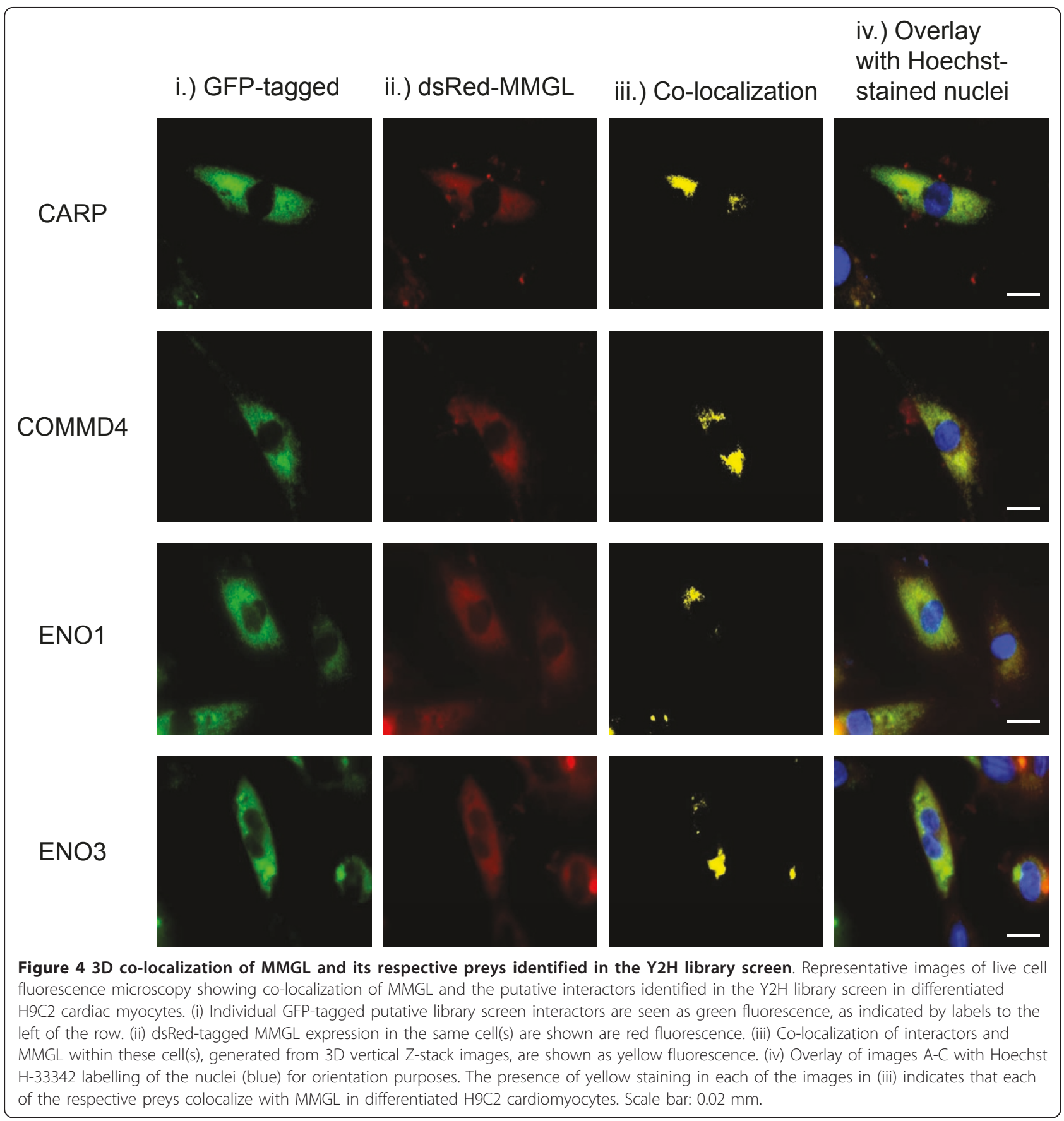


A)

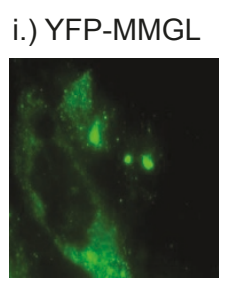

ii.) CTNI

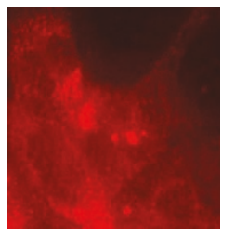

iii.) Co-localization iv.) cardiac actin

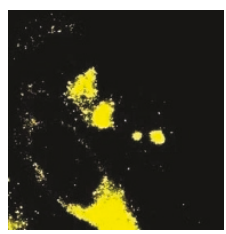

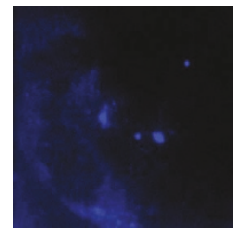

B)
i.) GFP-cTNI

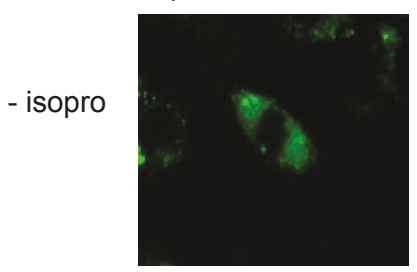

+ isopro

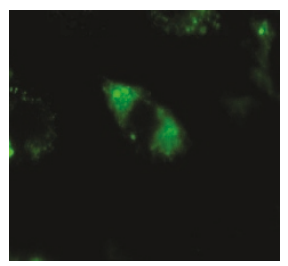

ii.) dsRed-MMGL
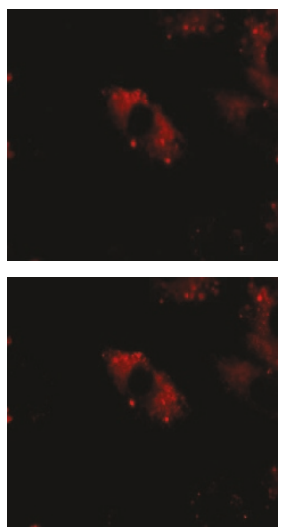

iii.) Co-localization
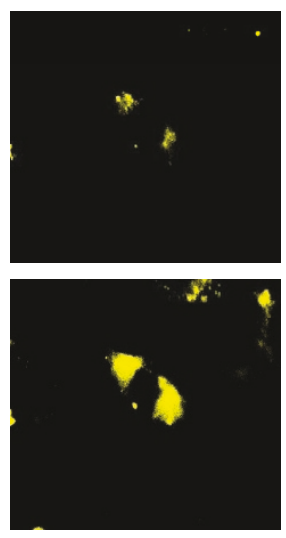

iv.) Overlay with Hoechst-stained nuclei
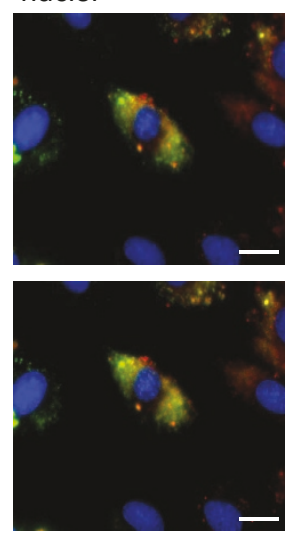

C)

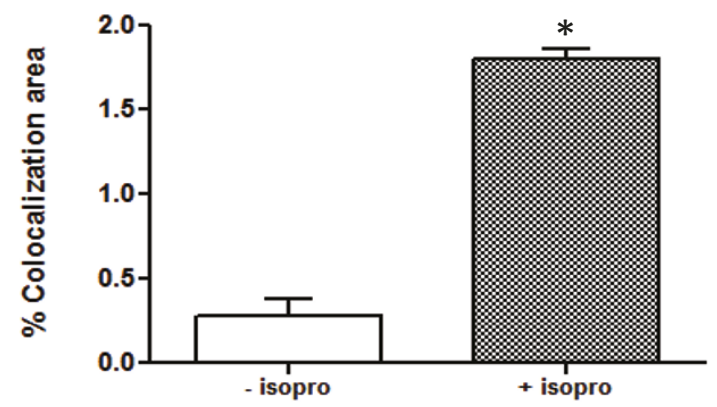

Figure 5 Co-localization increases between MMGL and PKA target upon $\boldsymbol{\beta}$-adrenergic stimulation. A. Representative image of live cell fluorescence microscopy showing co-localization of CTNI and MMGL isoform 4. Each panel represents a single frame of the 25 images that were captured for the vertical Z-stack. The first four panels show a single colour channel, while the image in the last panel shows an overlay of the four colour channels used. Column (iii) shows co-localization (yellow fluorescence) between dsRed-cTNI and YFP-MMGL, while column (iv) shows cardiac actin, a marker of the sarcomeric region. Scale bar: $0.02 \mathrm{~mm}$. B. Representative image of live cell fluorescence microscopy showing that co-localization of MMGL isoform 4 and cTNI increases under adrenergic stress. Each panel represents a single frame of the 25 images that were captured for the vertical Z-stack. Each of the first three columns shows a single colour channel, while the image in the last column shows an overlay of the four colour channels used. Column iii shows co-localization (yellow fluorescence) between dsRed-MMGL and GFP-cTNI in the absence (-isopro) and presence (+isopro) of the beta-adrenergic agonist, isoproterenol. The increase in intensity of yellow fluorescence in the second row demonstrates that co-localization levels of MMGL and cTNI had increased ten minutes after the addition of isoproterenol. Scale bar: $0.02 \mathrm{~mm}$. C. Quantification of co-localization shown in B shows that co-localization increased significantly $\left( \pm\right.$ SEM, $\left.{ }^{*} p<0.05, n=6\right)$ after the addition of isoproterenol. Change in co-localization was calculated using the CellR software and presented as a false colour image and percent co-localization as described by Loos et al., 2008 [29]. Abbreviations: isopro = isoproterenol. 


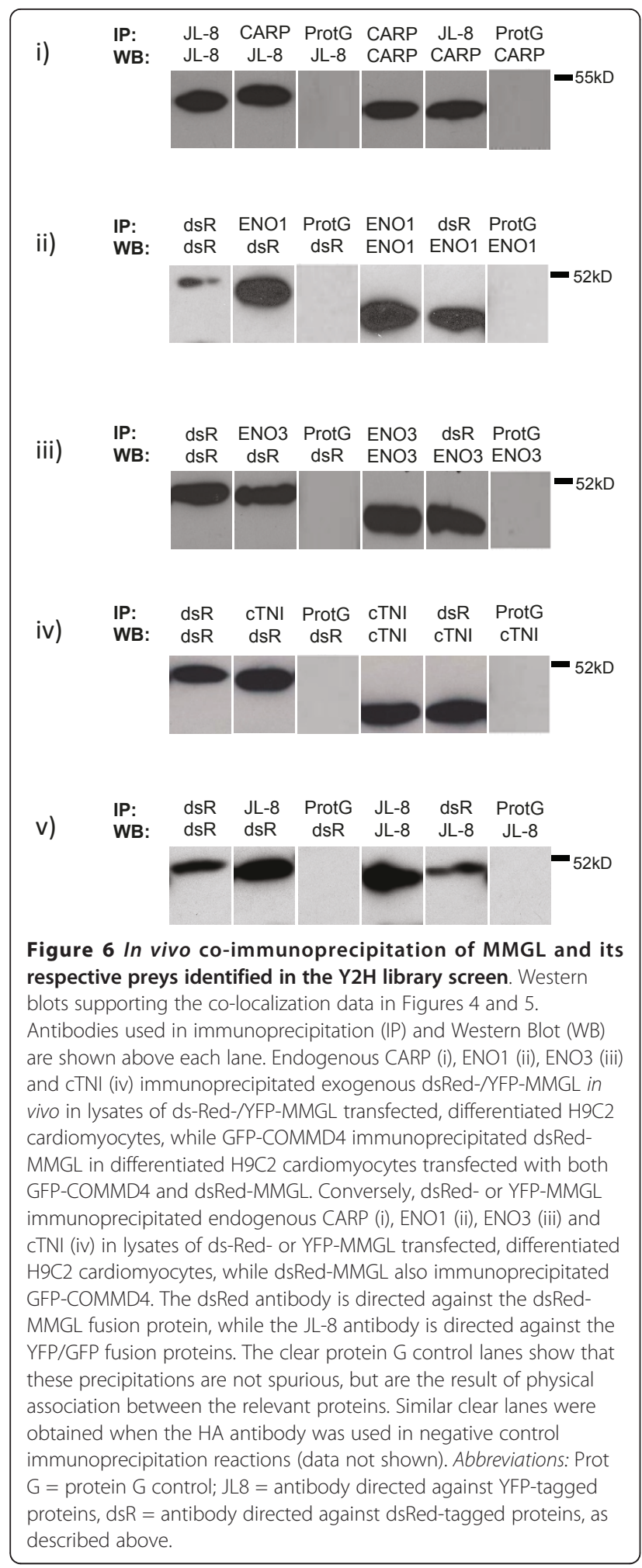

context of siRNA-mediated MMGL knockdown. Typically, interference with AKAP-functioning is commonly more noticeable on the target protein only after adrenergic stimulation [16], thus we also tested the effect of
MMGL knockdown in the presence of adrenergic stimulation. Of four siRNAs tested, Rn_RGD:708410_3_HP siRNA (MMGL 3) (Qiagen) was found to provide optimal knockdown of MMGL (80\%) in H9C2 cells (Figure 7A); thus MMGL 3 siRNA was used in subsequent experiments to silence MMGL gene expression.

Using Western blots of 2-dimensional IEF gels, we found that similar amounts of the mono- and diphosphorylated forms of cMyBPC were expressed in untreated $\mathrm{H} 9 \mathrm{C} 2$ cells, while lesser amounts of the unand trisphosphorylated forms, relative to the other isoforms, were present (Figure 7Bi, Ci). When these cells were exposed to increased $\mathrm{CaCl}_{2}$ and isoproterenol, to activate CaMK and PKA such that maximum phosphorylation of the MyBPC-motif is likely[7], the relative amount of the trisphosphorylated form increases markedly, while the relative amount of unphosphorylated cMyBPC decreased (Figure 7Bii, Cii). In untreated cells lacking MMGL, all four forms of cMyBPC are present, with amounts of mono- and diphosphorylated forms similar and exceeding the amounts of un- and trisphosphorylated forms, similar to what was seen with untreated, wild-type cells. However, adrenergic stimulation in the presence of MMGL knockdown resulted in very low expression of all isoforms (Figure 7Biv, Civ). This finding is compatible with previous findings of an inverse relationship between phosphorylation of $\mathrm{cMyBPC}$ and its proteolytic degradation, suggesting that phosphorylated cMyBPC is protected against proteolytic cleavage, whereas absence of phosphorylation results in increased degradation of the protein and reduced levels of cMyBPC in the cell $[17,18]$.

\section{Discussion}

Myomegalin has been characterized as a protein with the properties of a scaffold or structural protein that is expressed at high levels in skeletal and cardiac tissue, suggesting an important function in muscle, and which interacts with a cAMP-specific phosphodiesterase [13]. However, the precise function and interactions of this protein, and its five isoforms, have been largely unknown. We here describe how the smallest MMGL isoform, isoform 4, binds to known and predicted PKA targets in the cardiac myocyte, including some sarcomeric proteins, viz. cMyBPC, cTNI, ENO1, ENO3, CARP and COMMD4 (Tables 1 and 2). Moreover, we show that MMGL isoform 4 interacts with two regulatory subunits of PKA (Figure 3). Together these results describe MMGL isoform 4 as a novel sarcomeric AKAP, which, like mAKAP [14], is involved in assembling a PKA/PDE cAMP signalling module.

In addition to interacting with both types of regulatory subunits, viz. RI and RII, which qualifies MMGL isoform 4 as a dual-specific AKAP $[11,19]$. MMGL also meets the 
A:

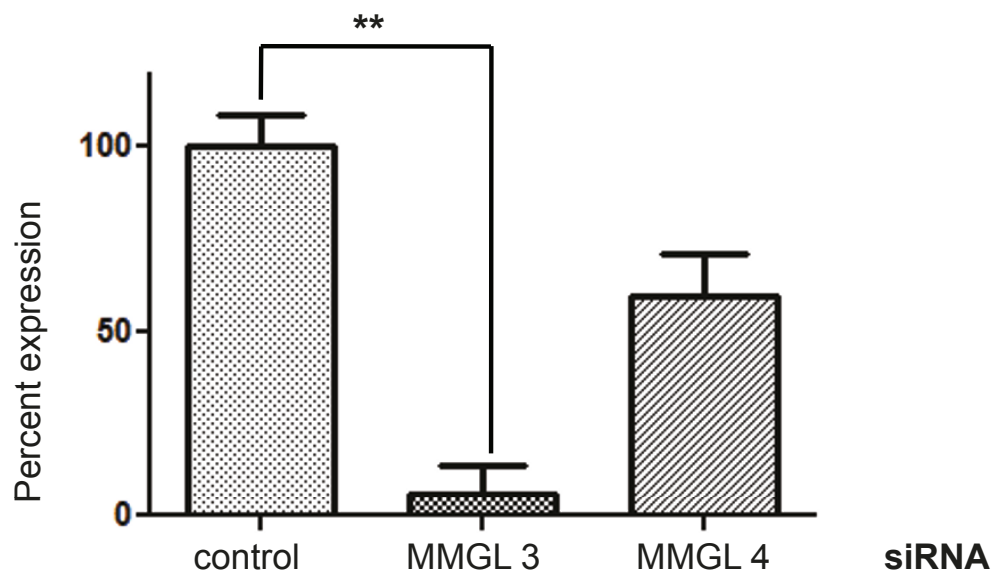

B)

(i)

MMGL SIRNA:

$\mathrm{CaCl}_{2}$, Isopro:

WB: JL-8
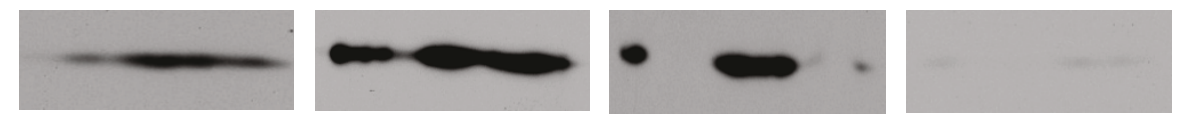

2
C)

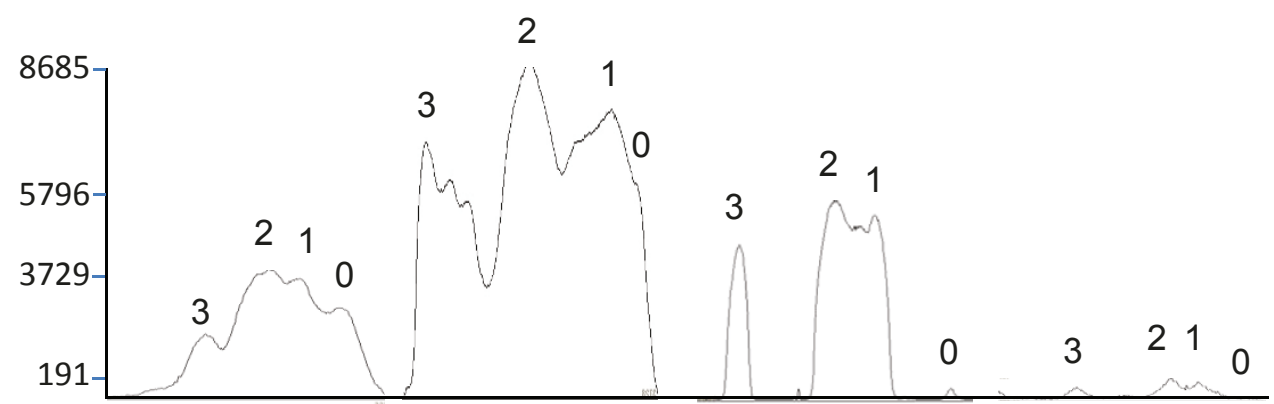

Figure 7 siRNA-mediated knockdown of MMGL leads to reduced levels of cMyBPC phosphorylation under adrenergic stimulation. A. Graph demonstrating that real-time quantification of MMGL CDNA transcribed from RNA extracted from cells transfected with either a nonsilencing control or a particular MMGL siRNA. Pde4dip Rn_RGD:708410_3_HP (MMGL 3) resulted in optimal knockdown of MMGL (80\%). B. Western blots of 2-dimensional IEF gels showing the expression of the four phosphorylation isoforms of GFP-cMyBPC in H9C2 cells (i) under non-stimulated conditions; (ii) under adrenergic stimulation, (iii) under non-stimulated conditions in the absence of MMGL (i.e. with MMGL knock-down) and (iv) under adrenergic stimulation in the absence of MMGL. C. Quantification of CMyBPC isoforms in the autoradiographs of the 2-dimensional IEF gels shown in (B), graphing the levels of the four phosphorylation isoforms ( $0=$ unphosphorylated; $1=$ monophosphorylated, 2 = diphosphorylated, 3 =trisphosphorylated). B and C(i) show that under non-stimulated conditions, levels of the mono- and diphosphorylated forms are similar and increased compared to the trisphosphorylated form. B and C(ii) show that under adrenergic stimulation, there is a relative increase in the trisphosphorylated form of CMyBPC and reduction of the non-phosphorylated form. B and C (iii) show that under non-stimulated conditions, ratios of the four forms of CMyBPC are similar to that in wild-type cells. B and C(iv) show that, upon adrenergic stress, knock-down of MMGL leads to a reduction in the levels of all CMyBPC phosphorylation forms. Abbreviations: JL8 = antibody directed against GFP-MyBPC. 
three other criteria for classification as an AKAP: By verifying its interaction with its proposed binding partners, we demonstrate that the targeting domains contained within the proposed AKAP participates in protein-protein interactions [11]. Further, it has previously been shown that MMGL is a phosphodiesterase 4D-interacting protein [13], thus, it meets the criterion for being able to coordinate multiple signalling pathways by anchoring additional signalling enzymes $[11,20]$. Lastly, we have shown in other $\mathrm{Y} 2 \mathrm{H}$ screens that cMyBPC also binds to COMMD4 (unpublished results), here shown to be a MMGL interactor, while COMMD4 itself also binds to ENO1 and SNX3 (unpublished results). This strongly suggests that MMGL is part of a larger, multiprotein unit $[11,20]$, and that MMGL isoform 4 may function as a vital link in signaling between upstream activators and numerous downstream targets [21].

Co-compartmentalization of both PKA and PDE4D is crucial for maintaining specificity of adrenergic signaling, and for maintaining contractility in cardiac cells [16]. We here established an important and novel link between PKA and PDE4D co-compartmentalization at the sarcomere level, and cMyBPC phosphorylation, and hence, regulation of cardiac contraction. The mechanism of docking of PKA to cMyBPC for phosphorylation of the MyBPC motif has previously not been elucidated; this study strongly suggests that MMGL isoform 4 anchors PKA to the N-terminal region, viz. C1-C2, of cMyBPC. The interaction between MMGL isoform 4, PKA, PDE4D and the N-terminal region of cMyBPC therefore sheds light on how second messenger responses are regulated in this particular part of the sarcomere.

We also found that $\beta$-adrenergic stimulation led to greater co-localization of myomegalin with both cMyBPC and cTNI in live cardiomyocytes, as evidenced by the increase in yellow staining during fluorescence microscopy in Figures 1 \&5, respectively. Thus, although MMGL is apparently present within the sarcomere under normal conditions, this implies that under adrenergic stimulation, and consequential increased intracellular levels of cAMP, PKA is dynamically recruited by MMGL isoform 4 to distinct sarcomeric locations. This translocation of MMGL to the sarcomeric region is therefore compatible with a mechanism that would lead to increased phosphorylation of cMyBPC and cTNI, which is known to form part of the cardiac cellular stress response that leads to increased cardiac contraction [22]. Furthermore, given MMGL's known interaction with PDE4D [13], the mechanism for termination of the second messenger response, by degrading cAMP, would also be on site; lower levels of cAMP may then cause this multiprotein complex to dissociate again.
The knockdown studies of MMGL further suggests that MMGL not only acts as an AKAP at the MyBPC motif, but by implication plays a role in cardioprotection during adrenergic signaling. Although, in the presence of MMGL, all phosphorylation isoforms of cMyBPC are expressed well in $\mathrm{H} 9 \mathrm{C} 2$ cells, and the level of the trisphosphorylated cMyBPC is increased in such cells under conditions of $\beta$-adrenergic stimulation as is to be expected, knockdown of MMGL under adrenergic conditions dramatically reduced cMyBPC expression (Figure 7). The latter finding suggests that when MMGL expression is reduced, cMyBPC phosphorylation is hindered, rendering the protein vulnerable to cleavage by proteases and reducing cMyBPC protein levels in the cell, as described by others $[17,18,23]$. Typically, annulment of the effect of an AKAP is commonly more noticeable on the target protein only after adrenergic stimulation: for instance, the studies of McConnell et al. (2009) [24] and Fink et al. (2001) [16] showed no significant difference in the level of phosphorylation of key cardiac proteins between wild-type cardiomyocytes and those in which AKAP function had been impaired with the Ht31 peptide. However, upon isoproterenol stimulation, dramatically decreased levels of PKA phosphorylation of all these proteins was observed in Ht31-transfected cells compared to isoproterenol-stimulated wild-type cells. Fink et al. (2001) [16] also demonstrated that AKAPs regulate phosphorylation of these PKA targets, including cTNI and cMyBPC, in response to $\beta$-adrenergic stimulation, although it has been unknown which AKAP/s are responsible for the phosphorylation of these two proteins up until our study. Thus, it appears that MMGL is an essential part in the $\beta$-adrenergic pathway leading to trisphosphorylation of $\mathrm{cMyBPC}$ and protection of the protein against degradation and normal sarcomeric integrity, which in turn is essential for normal physiological cardiac function, as well as cardioprotection during ischemia-reperfusion injury [25].

The subcellular localization and functions of some of the putative PKA targets identified via the $\mathrm{Y} 2 \mathrm{H}$ library screen suggest that MMGL may act as AKAP in regions outside the sarcomere as well. Although some of the identified interactions, such as those with $\mathrm{cMyBPC}$ and cTNI, certainly occur within the sarcomere, associations with the other identified interactors (Table 2) do not necessarily occur within the sarcomere, nor do they necessarily all occur concurrently. In fact, a multiprotein subunit in the sarcomere consisting simultaneously of all identified MMGL-ligands would most likely sterically hinder crossbridge formation, and is therefore improbable. However, interaction of MMGL with proteins such as COMMD4, CARP, ENO1 and ENO3 may facilitate control of efficient PKA phosphorylation of these 
proteins; the protein-protein interactions and/or activation of these proteins may thus be regulated.

Although this study prioritized investigation of the interaction between the $\mathrm{N}$-terminal of cMyBPC and MMGL, the other putative interactions of this region of cMyBPC should be further explored. The absence of cMyBPC as a prey in the MMGL Y2H library screen is most likely explained by the known absence of cDNAs representing the $\mathrm{N}$-terminals of large proteins in oligo $\mathrm{dT}$-primed libraries, while the absence of PRKAR1A and PRKAR2A may relate to the stringency of selection during heterologous bait-matings during this $\mathrm{Y} 2 \mathrm{H}$ screen [26].

Interaction between myomegalin, PKA and cMyBPC or cTNI is also relevant to understanding of HCM patho-aetiology, as both of the latter proteins cause $\mathrm{HCM}$ when defective. It is known that point mutations in the $\mathrm{C} 1-\mathrm{C} 2$ region and in the MyBPC motif lead to HCM [3,27]; a potential mechanism for this may be involve disruption of binding between MMGL and cMyBPC. Such disruption would have consequences for PKA-phosphorylation of the cMyBPC motif (with implications for regulation of cardiac contractility), implying a poison-peptide mechanism underlying disease, as well as maintenance of sufficient cMyBPC levels within the sarcomere, particularly under conditions of adrenergic stress, implying a haplo-insufficiency mechanism. Point mutations in cTNI may have a similar patho-aetiology. It could be further speculated that, mutations in MMGL could similarly lead to inadequate binding of PKA and/or its sarcomeric partners, which might affect cMyBPC or cTNI phosphorylation and hence affect adaptation of cardiac contractility to adrenergic stimulation. Future studies could therefore consider MMGL as either a candidate causal gene or a potential modifier gene for HCM.

\section{Conclusions}

This study shows that myomegalin isoform 4 is a novel sarcomeric AKAP, which forms part of a multiprotein complex that functions in CAMP signalling. It is particularly relevant to phosphorylation of cMyBPC and cTNI, and therefore, is of significance in the regulation of cardiac contractility in both health and disease.

\section{Methods \\ Plasmid constructs $\mathrm{Y} 2 \mathrm{H}$ constructs}

The region of MYBPC3 encoding domains C1-C2 was PCR-amplified from a MYBPC3 cDNA clone (kind gift of Prof Hugh Watkins, Oxford University). PCR-based site-directed mutagenesis, as previously described by Elliott et al. [28], was then used to generate a PCR fragment representing domains $\mathrm{C} 1-\mathrm{C} 2$ in which the codons for serines within all three phosphorylation sites of the cMyBPC motif were mutated to encode glutamic acids to mimic the trisphosphorylated state (PPP). The fulllength cDNA from MMGL isoform 4 was amplified from a commercial construct, in vector pdEYFP-C1 (imaGenes $\mathrm{GmbH}$ ). These fragments were individually cloned into the NdeI and EcoRI restriction sites inframe with GAL4BD in the $\mathrm{Y} 2 \mathrm{H}$ bait yeast expression vector pGBKT7 (Clontech) for use in the respective $\mathrm{Y} 2 \mathrm{H}$ library screens or in $\mathrm{Y} 2 \mathrm{H}$-based direct protein-protein experiments.

The cDNA of the two PKA regulatory isoforms (PRKAR1A and PRKAR2A) were PCR amplified from a cardiac cDNA library (Clontech). These fragments were cloned into the $B a m \mathrm{HI}$ and $X h o \mathrm{I}$ restriction sites or the $\mathrm{NcoI}$ and BamHI sites, respectively, of the $\mathrm{Y} 2 \mathrm{H}$ prey vector pACT2 (Clontech).

Integrity of insert sequences, reading frame and cloning sites were verified by means of bi-directional sequencing, after which pGBKT7-PPP and pGBKT7MMGL were transformed into $S$. cerevisiae strain AH109, and pACT2-R1A and pACT2-R2A into strain Y187 (Clontech).

\section{Constructs used for verification analyses}

The cDNAs from the putative interactors of MMGL isoform 4 identified in the $\mathrm{Y} 2 \mathrm{H}$ library screen viz. TNNI3, CARP, COMMD4, ENO1 and, ENO3, as well as PRKAR $1 A$ and PRKAR2A, were PCR amplified and cloned into the pGFP2-C1 fluorescent vector (BD Bioscience). MMGL was further subcloned from the pGBKT7-MMGL construct into the $\mathrm{pDs}$-Red-C1 vector (dsRed-MMGL) (BD Bioscience). The integrity of the cloning sites, reading frames and all interactor sequences were verified by bi-directional sequencing. These constructs were subsequently used in 3D in vivo co-localization and in vivo co-immunoprecipitation analyses.

\section{Y2H library screening}

A S. cerevisciae Y187 pre-transformed human MATCHMAKER $^{\mathrm{TM}}$ cardiac CDNA library constructed in pACT2 (BD Bioscience) was mated with the AH109 strain transformed with pGBKT7-PPP and the library screen performed according to manufacturer's instructions. Clones that expressed all three reporter genes, HIS3, ADE2, and $M E L 1$, were further analyzed. An interaction-specificity test was used to identify preys that did not activate reporter genes in the presence of the following heterologous baits: pGBKT7-C5 (encoding Igl domain C5 of cMyBPC), pGBKT7-53 (encoding murine p53) and unrecombined pGBKT7. Prey plasmids interacting specifically with PPP were sequenced using a vector specific primer, and in-frame ORF sequences analyzed via BLASTN and BLASTP http://ncbi.nlm.nih.gov/blast to 
assign identity. Literature and public database searches were used to prioritize putative ligands according to function and subcellular localization. A Y2H library screen with pGBKT7-MMGL was subsequently similarly performed.

\section{Direct $\mathrm{Y} 2 \mathrm{H}$ protein-protein interaction assay}

pGBKT7-MMGL in yeast strain AH109 was mated individually with pACT2-R1A and pACT2-R2A in yeast strain Y187 to determine whether the PKA regulatory subunits interacts directly with MMGL. A single colony of pGBKT7-MMGL in AH109 was taken from appropriate selection plates and suspended with a single colony of pACT2-R1A or -R2A in YPDA media overnight. Mating mixes were plated onto solid growth medium lacking leucine (Leu) and tryptophan (Trp). Following an incubation period of four days, the colonies on each plate were transferred to medium lacking Leu, Trp and histidine (His), and incubated five days. Surviving colonies were finally transferred to medium lacking Leu, Trp, His and adenine (Ade) and growth was assessed on day 4 and 7 . Control matings were included those of pGBKT7-MMGL with pACT2-cTNI(+), pACT2-TUSC4 (-) and empty pACT2(-).

\section{Antibodies}

Anti-HA, anti-Myc and anti-CARP antibodies were purchased from Santa Cruz Biotechnology. Living colours $^{\mathrm{TM}}$ anti-dsRed (directed against dsRed-tagged proteins) and JL-8 (directed against GFP and YFP-tagged proteins) antibodies were purchased from Clontech and anti- $\beta$ actin antibody from Cell Signaling Technology. AntiENO1, -ENO3, -PRKAR1A, -PRKAR2A and -cTNI antibodies were purchased from Abnova.

\section{Cell culture and transfection}

$\mathrm{H} 9 \mathrm{C} 2$ cells were maintained at $37^{\circ} \mathrm{C}$ and $5 \% \mathrm{CO} 2$ in Dulbecco's modified Eagle's medium, supplemented with $10 \%$ fetal bovine serum, $100 \mu \mathrm{g} / \mathrm{ml}$ penicillin and 100 $\mu \mathrm{g} / \mathrm{ml}$ streptomycin. For in vivo co-immunoprecipitation, approximately 4 million $\mathrm{H} 9 \mathrm{C} 2$ cells were seeded per $135 \mathrm{~mm}$ petri dish, and were transfected once 70$80 \%$ confluency was reached. Genejuice ${ }^{\circledR}$ (Novagen) was used for transfections according to manufacturer's instructions. For 3D in vivo co-localization, approximately 20000 - $30000 \mathrm{H} 9 \mathrm{C} 2$ cells were seeded per well in 8-well borosilicate coverglass chambers (Nunc), and transfected after 70-80\% confluency have been reached using Lipofectamine ${ }^{\mathrm{TM}}$ (Invitrogen) according to manufacturer's recommendations. Cells were differentiated into myotubes $24 \mathrm{~h}$ after transfection by changing the growth media to differentiation media (Dulbecco's modified Eagle's medium, 1\% horse serum, $100 \mu \mathrm{g} / \mathrm{ml}$ penicillin and $100 \mu \mathrm{g} / \mathrm{ml}$ streptomycin). Fresh differentiation media was added every $48 \mathrm{~h}$ until cells were fully differentiated.

\section{Three-dimensional in vivo co-localization}

Prior to image acquisition, the differentiation media was removed from the co-transfected, differentiated $\mathrm{H} 9 \mathrm{C} 2$ cells and replaced with culture media containing a 1:200 dilution of Hoechst H-33342 nucleic acid stain (Sigma). Cells in three wells co-transfected with GFP-MyBPC/ dsRed-MMGL were photographed using an Olympus IX 81 motorised inverted microscope (Olympus); after photography, cells were treated with $0.1 \mu \mathrm{M}$ isoproterenol in order to stimulate phosphorylation of the MyBPC motif [7] and the same cells photographed again, to monitor changes in co-localization upon adrenergic stimulation. CellR software was used to perform image analysis. Z-stacks were done in order to co-localize the tagged proteins in three dimensions. Double-labelled images, utilizing the co-transfected samples, were obtained at different focal planes which were processed by the CellR software to determine co-localization. A $60 \mathrm{X}$ oil immersion objective was used to collect image stacks at $0.26 \mu \mathrm{m}$ intervals in the plane. Subsequently, each co-localized image was created from the average of 25 frames. Change in co-localization was calculated using the CellR software and presented as a false colour image and percent co-localization as described by Loos et al., 2008 [29]. Changes in co-localization of MMGL isoform 4 and cTNI were obtained in a similar manner.

\section{In vitro protein transcription and translation}

The cDNA encoding the native as well as the trisphosphorylated mimic of the $\mathrm{C} 1-\mathrm{C} 2$ region of $\mathrm{CMyBPC}$ and the full-length MMGL isoform $4 \mathrm{cDNA}$ were PCR amplified to generate fragments that incorporates the T7 promoter and Myc- or HA-epitope tags, respectively. The TnT Quick Coupled Transcription/Translation System (Promega) was then used to transcribe and translate these PCR products into Met-35S-radiolabelled proteins, according to manufacturer's instructions.

\section{In vitro co-immunoprecipitation}

Five microlitres of the respective 35S-labelled HAMMGL and Myc-C1-C2 were mixed and incubated for $1 \mathrm{~h}$ at room temperature. One microlitre of anti-HA or anti-Myc antibody $(5 \mu \mathrm{g} / \mathrm{ml})$ was added followed by incubation of $1 \mathrm{~h}$. Ten microlitres of pre-washed protein G agarose (Kirkegaard \& Perry Laboratories) were subsequently added, together with $135 \mu \mathrm{l}$ Co-IP buffer [ 5 $\mathrm{mM}$ phosphate-buffered saline (PBS), $5 \mu \mathrm{g} / \mathrm{ml}$ aprotinin, $0.5 \mathrm{mM}$ PMSF, $100 \mathrm{mM}$ DTT, $1 \%$ Tween-20]. Incubation of $1 \mathrm{~h}$ at $4^{\circ} \mathrm{C}$ followed on a rotating device. Centrifugation followed for $30 \mathrm{~s}$ at $3000 \mathrm{~g}$, after which the pellet was washed five times with Tris-buffered saline 
with Tween-20 [20 mM Tris- $\mathrm{HCl}, \mathrm{pH} 7.5,150 \mathrm{mM}$ $\mathrm{NaCl}, 1 \%$ Tween-20]. Single immunoprecipitations were performed in the same way, using the same volumes of either bait or prey transcripts as were used in the coimmunoprecipitation reactions. Samples were electrophoresed on a $20 \%$ SDS-polyacrylamide gel, and protein bands were detected by autoradiography.

\section{In vivo co-immunoprecipitation}

$\mathrm{H} 9 \mathrm{C} 2$ cells transfected with the appropriate constructs were harvested $48 \mathrm{~h}$ following transfection, centrifuged at $3000 \mathrm{rpm}$ for $3 \mathrm{~min}$ and the pellet washed with PBS. Two hundred microlitres of passive lysis buffer (PLB) [0.5 M EDTA, $1 \mathrm{M}$ NaVO4, $10 \mathrm{mM}$ Nappi, $1 \mathrm{M}$ HEPES, $5 \mathrm{M} \mathrm{NaCl}, 1 \%$ TritonX, protease inhibitors and phenylmethylsulfonyl fluoride (PMSF)] was added to each sample. Samples were then incubated on ice for 30 min followed by centrifugation at $14000 \mathrm{rpm}$ and the supernatants collected. Lysate concentration was determined via a Bradford assay, and the volumes equalized to $200 \mu \mathrm{l}$ by adding PLB containing protease inhibitors and PMSF with a final concentration of $200 \mu \mathrm{g} / \mu \mathrm{l}$. Lysates were pre-cleared by adding $20 \mu$ Protein G agarose beads (KPL) and incubating samples at $4{ }^{\circ} \mathrm{C}$ for $2 \mathrm{~h}$ on a rotating device. Fluorescent-tagged or endogenous proteins were immunoprecipitated from lysates using 1-2 $\mu \mathrm{g}$ of the appropriate antibody, as indicated in Figure $2 \mathrm{c}$ and 5. Following overnight incubation, $60 \mu \mathrm{l}$ of protein $\mathrm{G}$ agarose beads was added to each sample, and incubated at $4^{\circ} \mathrm{C}$ for $2 \mathrm{~h}$ on a rotating device. Afterwards, immunoprecipitates were washed $5 \mathrm{X}$ in cold PLB containing protease inhibitors and PMSF, followed by resuspension in SDS loading buffer, and boiling prior to SDS-PAGE and subsequent Western blotting. Negative controls included samples in which protein $G$ only was used in the absence of antibody, samples in which the HA-antibody was used as a negative control-antibody, and samples that had not been transfected with the dsRed-MMGL construct.

\section{RNA interference}

The effect of siRNA transfection on myomegalin mRNA expression using different PDE4DIP siRNAs (Qiagen) was determined and optimized by a 2-step Q-RT-PCR using the Corbett Rotorgene system as follows: Approximately $4 \times 10^{4} \mathrm{H} 9 \mathrm{C} 2$ cells were seeded per well of an 8well chamber slide, and siRNA transfected when cells reached 50-60\% confluency, using HiPerFect transfection reagent (Qiagen) as per manufacturer's instructions. Total RNA extraction followed after 24 hours using the RNeasy Plus Mini kit (Qiagen) as per manufacturer's instructions. cDNA was subsequently transcribed using the Quantitect Reverse Transcription kit (Qiagen) as per manufacturer's instructions. The Quantifast SYBR green
PCR kit (Qiagen) was then used to perform a real-time quantification of cDNA transcribed from the extracted RNA with or without non-silencing control (NSC) or PDE4DIP siRNAs. PDE4DIP levels were quantified with reference to three rodent reference genes (transferring receptor - TRFR, glyceraldehydes-3-phosphate dehydrogenase - GAPDH and heat shock protein $1 \beta-H s p 1 \beta$ ) selected from a panel of 6 commonly used housekeeping genes.

The Genomewide designed non-validated Rn_RGD:708410_3_HP siRNA (Qiagen) resulted in the lowest MMGL gene expression quantification levels, and was used in subsequent experiments. Confluent $\mathrm{H} 9 \mathrm{C} 2$ cells were transfected with GFP-tagged cMyBPC using Genejuice $^{\mathbb{R}}$ (Novagen). These cells were then transfected after 24 h with $10 \mathrm{nM}$ PDE4DIP Rn_RGD:708410_3_HP siRNA using HiPerFect Transfection Reagent (Qiagen); control cells were not transfected with siRNA. For adrenergic stimulated cells, cells were treated with $65 \mathrm{mM}$ $\mathrm{CaCl} 2$ for $10 \mathrm{~min}$ at $24 \mathrm{~h}$ post-transfection, followed by a $0.1 \mu \mathrm{M}$ isoproterenol treatment for another $10 \mathrm{~min}$. All cells were then lysed (as done for in vivo co-immunoprecipitation) and concentrations determined via Bradford assays, and all volumes equalized to $200 \mu \mathrm{l}$ by adding PLB containing protease inhibitors and PMSF with a final concentration of $200 \mu \mathrm{g} / \mu \mathrm{l}$. A non-denaturing immunoprecipitation of GFP-cMyBPC from the lysate followed using $1 \mu \mathrm{g}$ of the JL- 8 antibody with the Dynabeads ${ }^{\circledR}$ Protein G and DynaMag ${ }^{\mathrm{TM}}-2$ system (Invitrogen) as per manufacturer's instructions. Isoelectric focusing of the GFP-cMyBPC immunoprecipitates followed to separate the four possible phosphorylation isoforms of cMyBPC.

\section{Isoelectric focusing}

For the first dimension separation, the GFP-tagged cMyBPC immunoprecipitates were suspended in ReadyPrep 2-D Rehydration buffer 1 (Bio-Rad) containing Bio-lite pH 3-10 buffer (Bio-Rad) to a final volume of $200 \mu \mathrm{l}$. The protein/rehydration buffer mix was then applied to a pH 4-7 $(11 \mathrm{~cm})$ immobilized $\mathrm{pH}$ gradient (IPG) strip (Bio-Rad). Rehydration of the strip followed for 12 hours at room temperature. Afterwards, IEF was done under the following conditions: $8000 \mathrm{~V}$ for 20 $\mathrm{min}, 8000 \mathrm{~V}$ for 2 hours and $8000 \mathrm{~V}$ for $40000 \mathrm{~V}$ hours at $21^{\circ} \mathrm{C}$ (Protean IEF Cell, Bio-Rad). Strips were stored at $-80^{\circ} \mathrm{C}$ after IEF until required.

For 2-dimensional gel electrophoresis (2-DE), IPG strips were equilibrated by incubating the strips in equilibration buffer $(0.375 \mathrm{M}$ tris- $\mathrm{HCl}, 6 \mathrm{M}$ urea, $20 \%$ glycerol, 2\%, SDS) containing DTT (Sigma-Aldrich) for 15 min and then in equilibration buffer containing iodoacetamide (Sigma-Aldrich) for $15 \mathrm{~min}$ shaking at room temperature. Following equilibration, the IPG strips 
were placed on top of a Criterion XT $12 \%$ bis-tris precast gel (Bio-Rad) and sealed with $0.5 \%$ agarose (BioRad). Electrophoresis of the second dimension separation was done under constant voltage $(200 \mathrm{~V})$ for 1-2 hours in 5\% XT-MOPS running buffer (Bio-Rad). Gels were then transferred to a nitrocellulose membrane and subsequently western blotted with the JL- 8 antibody.

\section{Acknowledgements}

This work was funded by the Wellcome Trust (International Senior Research Fellowship fellowship GR073610MA to JCM-S)

\section{Author details}

'US/MRC Centre for Molecular and Cellular Biology, Department of Biomedical Sciences, University of Stellenbosch, South Africa. ${ }^{2}$ Central Analytical Facility, Department of Physiology, University of Stellenbosch, South Africa.

\section{Authors' contributions}

GMU carried out the MMGL Y2H direct protein-protein interactions and library screen, cell culture, 3D in vivo co-localization assays, in vivo coimmunoprecipitations, RNA interference and isoelectric focusing, and drafted the manuscript. AR carried out the CMyBPC-PPP Y2H library screen and in vitro co-immunoprecipitations. BL assisted with operating the Olympus IX 81 inverted microscope and CellR software. CJK assisted with interpretation of data and technical assistance. LJK and JM carried out cell culture and optimized siRNA transfections and MMGL knockdown. JR and JM assisted with siRNA and western blot optimization. JCM-S conceived of the study, acquired funding, participated in its design, coordination and interpretation of data, and revised and approved the final manuscript. All authors read and approved the final manuscript, and there are no conflicts of interest.

Received: 20 September 2010 Accepted: 10 May 2011

Published: 10 May 2011

\section{References}

1. Gautel M, Zuffardi O, Freiburg A, Labeit S: Phosphorylation switches specific for the cardiac isoform of myosin binding protein-C: a modulator of cardiac contraction? EMBO J 1995, 14:1952-1960.

2. Garvey JL, Kranias EG, Solaro RJ: Phosphorylation of C-protein, troponin I and phospholamban in isolated rabbit hearts. Biochem J 1988, 249:709-714.

3. Richard P, Charron P, Carrier L, Ledeuil C, Cheav T, Pichereau C, et al: Hypertrophic cardiomyopathy: distribution of disease genes, spectrum of mutations, and implications for a molecular diagnosis strategy. Circulation 2003, 107:2227-2232

4. Maron BJ, Gardin JM, Flack JM, Gidding SS, Kurosaki TT, Bild DE: Prevalence of hypertrophic cardiomyopathy in a general population of young adults. Echocardiographic analysis of 4111 subjects in the CARDIA Study. Coronary Artery Risk Development in (Young) Adults. Circulation 1995 92:785-789.

5. Levine R, Weisberg A, Kulikovskaya I, McClellan G, Winegrad S: Multiple structures of thick filaments in resting cardiac muscle and their influence on cross-bridge interactions. Biophys J 2001, 81:1070-1082.

6. Weisberg A, Winegrad S: Alteration of myosin cross bridges by phosphorylation of myosin-binding protein $\mathrm{C}$ in cardiac muscle. Proc Natl Acad Sci USA 1996, 93:8999-9003.

7. McClellan G, Kulikovskaya I, Winegrad S: Changes in cardiac contractility related to calcium-mediated changes in phosphorylation of myosinbinding protein C. Biophys J 2001, 81:1083-1092.

8. Pawson T, Scott JD: Signaling through scaffold, anchoring, and adaptor proteins. Science 1997, 278:2075-2080.

9. Wong W, Scott JD: AKAP signalling complexes: focal points in space and time. Nat Rev Mol Cell Biol 2004, 5:959-970.

10. Pawson T, Nash P: Protein-protein interactions define specificity in signal transduction. Genes Dev 2000, 14:1027-1047.

11. Colledge M, Scott JD: AKAPs: from structure to function. Trends Cell Biol 1999, 9:216-221
12. Schlender KK, Bean LJ: Phosphorylation of chicken cardiac C-protein by calcium/calmodulin-dependent protein kinase II. J Biol Chem 1991, 266:2811-2817.

13. Verde I, Pahlke G, Salanova M, Zhang G, Wang S, Coletti D, et al: Myomegalin is a novel protein of the golgi/centrosome that interacts with a cyclic nucleotide phosphodiesterase. J Biol Chem 2001, 276:11189-11198.

14. Dodge KL, Khouangsathiene S, Kapiloff MS, Mouton R, Hill EV, Houslay MD, et al: mAKAP assembles a protein kinase A/PDE4 phosphodiesterase cAMP signaling module. EMBO J 2001, 20:1921-1930.

15. Patel JR, Fitzsimons DP, Buck SH, Muthuchamy M, Wieczorek DF, Moss RL: PKA accelerates rate of force development in murine skinned myocardium expressing alpha- or beta-tropomyosin. Am J Physiol Heart Circ Physiol 2001, 280:H2732-H2739.

16. Fink MA, Zakhary DR, Mackey JA, Desnoyer RW, pperson-Hansen C, Damron DS, et al: AKAP-mediated targeting of protein kinase a regulates contractility in cardiac myocytes. Circ Res 2001, 88:291-297.

17. Decker RS, Decker ML, Kulikovskaya I, Nakamura S, Lee DC, Harris K, et al: Myosin-binding protein C phosphorylation, myofibril structure, and contractile function during low-flow ischemia. Circulation 2005, 111:906-912.

18. Sadayappan S, Osinska H, Klevitsky R, Lorenz JN, Sargent M, Molkentin JD, et al: Cardiac myosin binding protein C phosphorylation is cardioprotective. Proc Natl Acad Sci USA 2006, 103:16918-16923.

19. Wang L, Sunahara RK, Krumins A, Perkins G, Crochiere ML, Mackey M, et al: Cloning and mitochondrial localization of full-length D-AKAP2, a protein kinase A anchoring protein. Proc Natl Acad Sci USA 2001, 98:3220-3225.

20. Dodge K, Scott JD: AKAP79 and the evolution of the AKAP model. FEBS Lett 2000, 476:58-61.

21. Dodge-Kafka KL, Langeberg L, Scott JD: Compartmentation of cyclic nucleotide signaling in the heart: the role of A-kinase anchoring proteins. Circ Res 2006, 98:993-1001.

22. Chandra M, Dong WJ, Pan BS, Cheung HC, Solaro RJ: Effects of protein kinase A phosphorylation on signaling between cardiac troponin I and the N-terminal domain of cardiac troponin C. Biochemistry 1997, 36:13305-13311.

23. Sadayappan S, Gulick J, Klevitsky R, Lorenz JN, Sargent M, Molkentin JD, et al: Cardiac myosin binding protein-C phosphorylation in a \{beta\}myosin heavy chain background. Circulation 2009, 119:1253-1262.

24. McConnell BK, Popovic Z, Mal N, Lee K, Bautista J, Forudi F, et al: Disruption of protein kinase $\mathrm{A}$ interaction with A-kinase-anchoring proteins in the heart in vivo: effects on cardiac contractility, protein kinase A phosphorylation, and troponin I proteolysis. J Biol Chem 2009, 284:1583-1592.

25. Barefield D, Sadayappan S: Phosphorylation and function of cardiac myosin binding protein-C in health and disease. J Mol Cell Cardiol 2010, 48:866-875.

26. Adler V, Pincus MR, Minamoto T, Fuchs SY, Bluth MJ, Brandt-Rauf PW, et al: Conformation-dependent phosphorylation of p53. Proc Natl Acad Sci USA 1997, 94:1686-1691.

27. Erdmann J, Daehmlow S, Wischke S, Senyuva M, Werner U, Raible J, et al: Mutation spectrum in a large cohort of unrelated consecutive patients with hypertrophic cardiomyopathy. Clin Genet 2003, 64:339-349.

28. Elliott RJ, Bennet AJ, Braun CA, MacLeod AM, Borgford TJ: Active-site variants of Streptomyces griseus protease B with peptide-ligation activity. Chem Biol 2000, 7:163-171.

29. Loos B, Smith R, Engelbrecht AM: Ischaemic preconditioning and TNFalpha-mediated preconditioning is associated with a differential CPLA2 translocation pattern in early ischaemia. Prostaglandins Leukot Essent Fatty Acids 2008, 78:403-413.

doi:10.1186/1471-2121-12-18

Cite this article as: Uys et al:: Myomegalin is a novel A-kinase anchoring protein involved in the phosphorylation of cardiac myosin binding protein C. BMC Cell Biology 2011 12:18. 